\title{
How often are chaotic transients in spatially extended ecological systems?
}

\author{
Mukeshwar Dhamala ${ }^{a}$, Ying-Cheng Lai ${ }^{b, *}$, Robert D. Holt ${ }^{\mathrm{c}}$

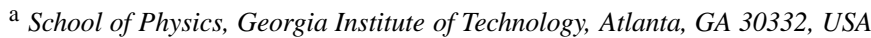 \\ ${ }^{\mathrm{b}}$ Departments of Mathematics, Electrical Engineering and Physics, Center for Systems Science and Engineering Research, \\ Arizona State University, Tempe, AZ 85287, USA \\ ${ }^{\mathrm{c}}$ Department of Systematics and Ecology, University of Kansas, Lawrence, KS 66045, USA
}

Received 23 October 2000; accepted 26 January 2001

Communicated by A.R. Bishop

\begin{abstract}
A traditional assumption in quantitative ecology is that the asymptotic state of the model determines what can be observed in the evolution of the system. It is suggested, however, that irregular transient behaviors may be more relevant than the long term behaviors. Here we investigate how often transient dynamics can be expected in spatially extended ecological systems. Our study suggests that although chaotic transient dynamics indeed exist, sustained dynamics may be more prevalent than transient ones due to the high dimensionality of such systems. (c) 2001 Published by Elsevier Science B.V.
\end{abstract}

PACS: 87.23.Cc; 87.23.-n; 05.45.In

Understanding the factors generating and maintaining the species diversity of ecological communities is one of the central goals of the ecological sciences. In the last several decades, ecologists have become increasingly aware of the importance of processes operating at large temporal and spatial scales in explaining patterns of species diversity in local communities [1]. This is particularly the case when considering guilds of organisms utilizing limited resources in similar ways. In spatially closed, homogeneous systems which dynamically settle into a point equilibrium, species sharing limited resources often show competitive exclusion. Because any given spatial location has a limited variety and quantity of resources, competi-

\footnotetext{
* Corresponding author.

E-mail address: yclai@ chaos1.la.asu.edu (Y.-C. Lai).
}

tion is a local process which tends to limit local species diversity. This local process in nature, however, interacts with other processes. For instance, if local dynamics do not achieve a point equilibrium (e.g., limit-cycle behavior in multispecies resource-consumer interactions [2]), consumer species can share resources yet still coexist. Many ecologists contend that patterns in species diversity cannot be understood without reference to nonequilibrial dynamics (for review, see [3]). Indeed, many ecologists now believe that space is the final frontier for addressing classical ecological problems $[4,5]$.

Simultaneous with this increasing emphasis on spatial dynamics, there has been an increasing interest in the potential existence and importance of chaotic dynamics in ecological systems. Although much of the general scientific interest in chaos is stimulated by simple ecological models [6], early evidence suggests 
that natural populations, despite their seemingly random fluctuations, do not have the kinds of parameter values needed for chaos to be observed [7], so variation in population size is believed to be due to externally imposed fluctuations (e.g., in the weather). This leads to a relative neglect of chaos by many ecologists. In recent years, there have been a reevaluation of this issue, and sophisticated analyses of dynamics in a wide range of natural communities, suggesting the presence of chaos (e.g., see $[6,8,9])$. Since chaotic behavior is typical in nonlinear dynamical systems [10], it is reasonable to search for causes of population fluctuations in underlying nonlinear dynamics.

The interplay between spatial dynamics and chaotic temporal dynamics is potentially of great importance in ecology. An important contribution is made by Hastings and Higgins [11] who report their finding of very long transient behaviors in spatially extended ecological models for a species with alternating reproduction and dispersal. They demonstrate, through numerical computations, that if the nonlinearity in the model is strong enough, then the time required to reach the asymptotic dynamics can approach thousands of generations - a time that is much longer than the time scale of significant environmental perturbations and therefore can be considered extremely long on ecological time scales of the species where the typical time scale of interest is tens or hundreds of years. Since the form of dynamics changes over long time scales, it is argued that transient dynamics of ecological models may be more relevant than long-term behavior [11]. This conclusion is quite surprising for the field of quantitative ecology because traditionally, ecological theory has been based on long-term behavior of ecological models, with stability analysis of the asymptotic state as primary tool $[8,12,13]$. The result is also important because it provides a possible explanation for the highly irregular dynamics of some species with pelagic larvae such as sea urchins [14] and Dungeness crab [15]. Long transients may also be responsible for the paradox of the plankton [16] or outbreaks of insects [17].

In view of the ecological significance of the existence of extremely long transients, it is of interest to understand the dynamical origin of the observed transients and, consequently, to establish how often we expect to observe complex (chaotic) transients in ecological systems. These are the aims of this Letter. A good understanding of the complex transient behavior is particularly important for spatially extended ecological systems, which are usually described by discretetime maps in continuous spatial domains and, hence, even numerical simulations of which can be highly nontrivial. For instance, one can ask whether the long transients observed by Hastings and Higgins are simply numerical artifacts. As we will describe in detail below, our careful integration of the Hastings-Higgins model using different numerical procedures, and our systematic analysis of bifurcation diagrams, Lyapunov spectrum, and lifetimes of chaotic behaviors over a wide range of parameter values, indicate that chaotic transients do occur in the model, but not so often. Care should also be exercised when claiming that the transients are extremely long because the transient lifetime depends on the choice of the parameters in the model and long chaotic transients are rare. In particular, say we fix a parameter region in which we expect chaotic behaviors to arise. We then ask: what is the probability that a randomly chosen parameter value yields chaotic transient behavior? We find that the probability can be quite low. In fact, for the same parameter setting as in Ref. [11], we find that, approximately, $5 \%$ of the parameter values yield chaotic transients, meaning that majority of the parameter values actually leads to sustained chaotic behavior (chaotic attractors). In a sense, our results are consistent with those of Hastings and Higgins, but we go beyond by establishing a clear dynamical picture for the observed transient behaviors and thus are able to address how often chaotic transients can occur in spatially extended ecological systems.

The Hastings-Higgins model describes the population dynamics of a biological species having features of intertidal or subtidal organisms with pelagic larvae along a coast. That is, the production of larvae is density dependent. After production, larvae are redistributed along the coast with a center at the point of release. Let the length of favorable habitat along the coast be $L=1$ and $x$ be the position along the coast: $0<x<1$. The dynamical variable of the model is the population $N(t, x)$ of the species along the coast, where $t$ is the discrete time variable (in year). The number of larvae produced at position $x$ in year $t$, $l(t, x)$, is then given by $[18,19]$

$l(t, x)=N(t, x) e^{r[1-N(t, x)]}$, 


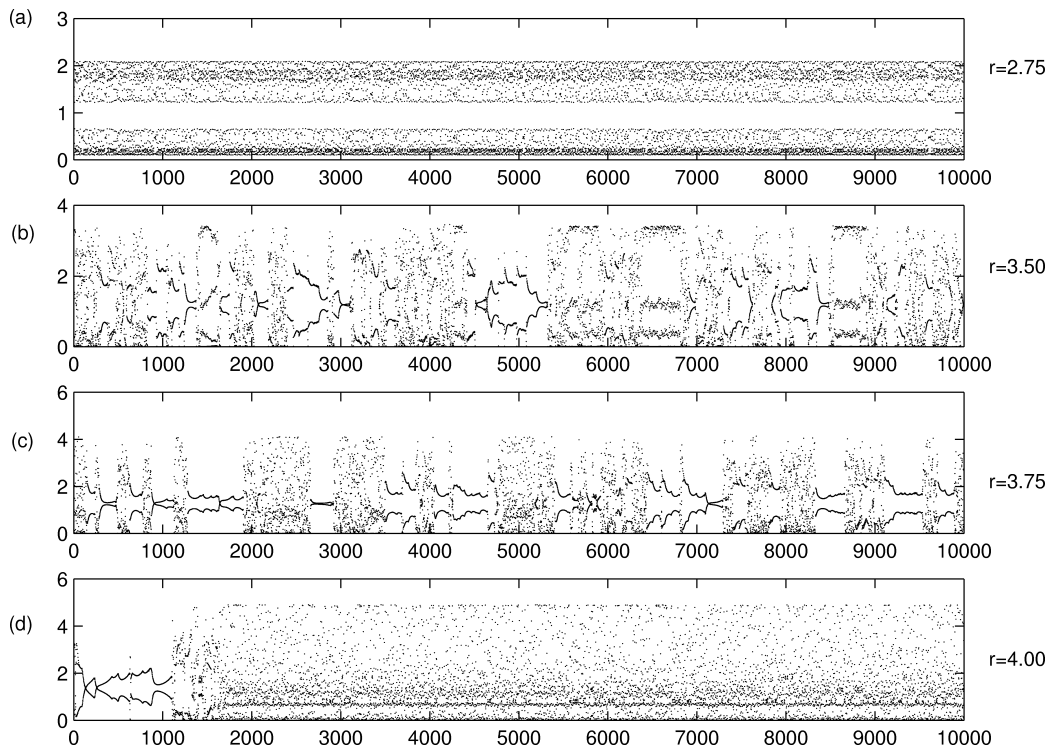

Fig. 1. For the Hastings-Higgins model, time series plots of local population $(N(t, 0.5))$ of species, growth rates being $r=2.75,3.50,3.75$, and 4.00, respectively, at $D=100$ (integrated by the Gaussian quadrature technique).

where $r$ is the growth rate of local population. Spatial interaction among the populations along the coast is caused by dispersal that follows reproduction. In particular, the number of individuals at point $x$ is given by the total number of larvae released about the position $x$ :

$N(t+1, x)=\int_{0}^{1} l(t, y) g(y, x) d y$,

where $g(y, x)$ is the probability that a larva released at location $y$ settles at $x$. Hastings and Higgins assumed the following Gaussian distribution for $g(y, x)$ :

$g(y, x)=\frac{e^{-D(y-x)^{2}}}{\sqrt{\pi / D}}$,

where $D$ is a parameter measuring the dispersal distance, or the "coupling strength" among populations in space. The boundary condition is such that larvae that disperse outside the region of interest $([0,1])$ are considered lost. Due to the spatial interaction and the boundary condition, the effective growth rate is not uniform in space. Eqs. (1)-(3) represent a spatially extended dynamical system that is discrete in time and continuous in space. To numerically evolve Eqs. (1)(3), we divide the unit interval $x \in[0,1]$ into 100 subintervals, distribute random initial populations at these sites, and at each time step, evaluate the integral Eq. (2) by using the Gaussian quadrature method [20]. To assure that the numerical results are reliable, we also utilize the Simpson's composite rule [20] to evaluate Eq. (2). Both integration methods have the precision of about $10^{-10}$, and they yield essentially the same spatial time series $N(t, x)$ that agree with those reported by Hastings and Higgins [11]. While long chaotic transients can indeed be observed, for example, at large $D$ values (say at $D=800$ ) [11], we notice that sustained chaotic behavior seems more common when $D$ is decreased. For instance, Fig. 1 shows the time trace of the population $N(t, 0.5)$ for different values of the growth rate $r$. There is apparently chaos for $r \geqslant 2.75$, which is consistent with the transition to chaos of the Moran-Ricker model which occurs, via a cascade of period-doubling bifurcations, at $r_{c} \approx 2.67$. The chaotic behavior observed for $r \geqslant 2.75$, however, seems sustained. We note that the spatial range of coupling is proportional to $1 / \sqrt{D}$. Therefore, large (small) $D$ values correspond to small (large) range of, or weak (strong), coupling. Numerical results obtained 



Fig. 2. (a) $P_{r}(\tau)$ versus $\tau$ and (b) $P_{D}(\tau)$ versus $\tau$. See text for details.

at a large number of $D$ values suggest that chaotic transients become rare as $D$ is increased (the coupling is reduced).

To quantitatively assess how often chaotic transients are, to the extent allowed by our available computational resource, we define the quantity $P(\tau)$, which is the probability that a chaotic transient of duration (lifetime) larger than $\tau$ is observed, where $P(0)=1$. Apparently, $P(\tau)$ is a nonincreasing function of $\tau$. Since sustained chaos has lifetime $\tau=\infty$, we expect $P(\tau)$ to reach a plateau, say, at $\tau=\tau_{c}$, as a function of $\tau$. The probability for sustained chaos is then $P\left(\tau_{c}\right)$ and that for transient chaos is $1-P\left(\tau_{c}\right)$. To numerically determine $\tau_{c}$ and $P\left(\tau_{c}\right)$, we fix an interval in the parameter space in which chaos can arise (say, after the accumulation of the period-doubling bifurcations), randomly choose a large number of parameter values in this interval, define a numerically achievable range for the lifetime (say, $0 \leqslant \tau \leqslant 10^{6}$ ), and compute the fraction of parameter values with lifetime larger than $\tau$. To numerically distinguish sustained chaos from the transient one, we make use of the largest Lyapunov exponent [21]. In particular, for a given parameter value, a trajectory is produced from a random set of initial conditions and the largest Lyapunov exponent $\lambda_{1}$ is computed as a function of time. At a given time $t$, if $\lambda_{1}$ is positive (negative), the trajectory is chaotic (nonchaotic). If $\lambda_{1}$ remains negative for a number of iterations, say 1000, the trajec-

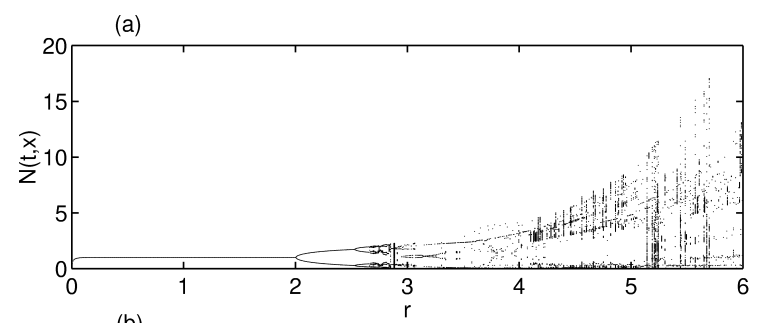

(b)



Fig. 3. Typical bifurcation diagrams of the Hastings-Higgins model: (a) $N(t, 0.5)$ versus $r$ and (b) $N(t, 0.5)$ versus $D$.

tory is considered having settled into a stable attractor. If this happens, the lifetime of the chaotic transient is taken to be the time during which $\lambda_{1}$ remains positive. Two sets of simulation results are shown in Figs. 2(a) and (b), where for Fig. 2(a), $D=800$ is fixed and 800 values of $r$ are chosen from the interval $r \in[3,6]$, and for Fig. 2(b), $r=4$ is fixed and 800 values of $D$ are chosen from $D \in[100,1400]$. We see from both figures that $P(\tau)$ plateaus at $\tau_{c} \sim 10^{4}$ and $P(\tau)$, where $\tau>\tau_{c}$, is about 0.955 , which indicates that the probability for observing transient chaos is less than $5 \%$ in the parameter space. The behavior depicted in Figs. 2(a) and (b) appears typical in parameter regions with chaos.

To understand why transient chaos is rare in the Hastings-Higgins model, we note that the local dynamics is governed by the Moran-Ricker map, which is bounded in the phase space. Thus, transient chaos occurs in periodic windows in the bifurcation diagram. In such a window, one typically finds a stable periodic attractor and a nonattracting chaotic set that leads to transient chaos. Figs. 3(a) and (b) show two typical bifurcation diagrams obtained from the HastingsHiggins model, where in Fig. 3(a), the asymptotic behavior of $N(t, 0.5)$ is plotted versus the growth rate $r$ for fixed $D=800$, while in Fig. 3(b), $N(t, 0.5)$ versus $D$ is shown for fixed $r=3.75$. These bifurcations indicate the existence of chaos, but they are qualitatively different from that of, say, the logistic map or 


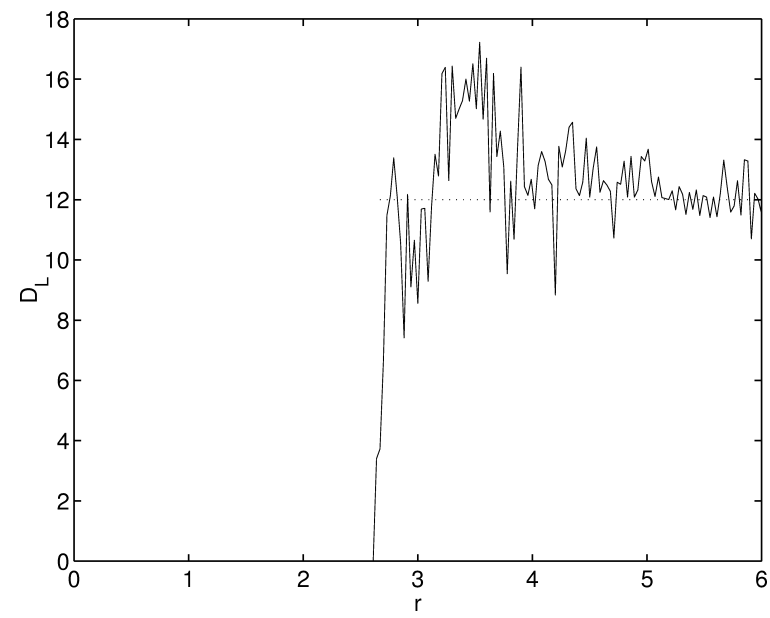

Fig. 4. For $D=800$, the Lyapunov dimension $D_{L}$ versus the growth rate $r$.

the Moran-Ricker map, in that there is a lack of structure of periodic windows, indicating the rareness of transient chaos. To give a more concrete argument, we recall that a low-dimensional chaotic system such as the Moran-Ricker map possesses an infinite number of periodic windows. Chaotic sets in the HastingsHiggins model, which is essentially a system of an infinite number of coupled Moran-Ricker maps, is most likely to be high-dimensional. To determine the dimension of the chaotic attractor, we compute the Lyapunov spectrum and make use of the Kaplan-Yorke formula to obtain the Lyapunov dimension $D_{L}$, which is conjected to the information dimension [22]. Fig. 4 shows, for $D=800, D_{L}$ versus $r$. We see that for $r>r_{c} \approx 2.67$, there is chaos but the dimensions of the chaotic attractor for most parameter values are larger than 10, resulting from Lyapunov spectra with, approximately, 6 positive exponents. By a mathematical conjecture proposed in Ref. [23], which states that there is only a finite set of bounded periodic windows if the number of positive Lyapunov exponents are larger than the number of independent parameters of the system, we see that periodic windows in the Hastings-Higgins model are finite in number because it has only two independent parameters. Computation of the Lyapunov dimension versus the coupling parameter for fixed growth rate $r$ yields a consistent result. Transient chaos is thus rare, compared with lowdimensional chaotic systems such as the logistic map or the Moran-Ricker map which possesses an infinite set of periodic windows.

In summary, we have analyzed the spatiotemporal chaotic dynamics for a class of spatially extended ecological model and present evidence that transient chaos is rare in such systems. Although our results do not contradict the previous claim that transient chaos can occur in ecological systems, we do wish to convey the message that one should be extremely cautious when claiming that transient chaos is more relevant than sustained chaos in spatiotemporal ecological systems.

\section{Acknowledgements}

This work is supported by NSF under Grant No. DMS-962659.

\section{References}

[1] M. Rosenzweig, Species Diversity in Space and Diversity, Cambridge University Press, 1995.

[2] R.A. Armstrong, R. McGehee, Amer. Natur. 115 (1980) 151.

[3] M. Huston, Biological Diversity: The Coexistence of Species on Changing Landscapes, Cambridge University Press, 1994.

[4] P. Kareiva, Ecology 75 (1994) 1.

[5] R. Ricklefs, D. Schluter, Species Diversity in Ecological Communities, University of Chicago Press, 1993.

[6] R.M. May, Nature 261 (1976) 459.

[7] M.P. Hassell, J.H. Lawton, R.M. May, J. Anim. Ecol. 45 (1976) 471

[8] A. Hastings, C.L. Hom, S. Ellner, P. Turchin, H.C.J. Godfray, Ann. Rev. Ecol. System. 24 (1993) 1.

[9] R.M. May, Bull. Amer. Math. Soc. 32 (1995) 291.

[10] J.-P. Eckmann, D. Ruelle, Rev. Mod. Phys. 57 (1985) 617.

[11] A. Hastings, K. Higgins, Science 263 (1994) 1133.

[12] R. May, Stability and Complexity in Model Ecosystems, Princeton University Press, Princeton, NJ, 1973.

[13] J. Roughgarden, R.M. May, S.A. Levin, Perspectives in Ecological Theory, Princeton University Press, Princeton, NJ, 1989.

[14] J.A. Estes, D.O. Duggins, G.B. Rathbun, Conserv. Biol. 3 (1989) 252.

[15] A.A. Berryman, Oikos 62 (1991) 106.

[16] G.E. Huschinson, Amer. Natur. 95 (1961) 137.

[17] P. Barbosa, J.C. Shultz, Insect Outbreaks, Academic Press, San Diego, CA, 1987.

[18] P.A.P. Moran, Biometrics 6 (1950) 250.

[19] W.E. Ricker, J. Fish. Res. Bd. Can. 11 (1954) 559. 
[20] W.H. Press, B.P. Flannery, S.A. Teukolsky, W.T. Vetterling, Numerical Recipes, Cambridge University Press, 1992.

[21] G. Benettin, L. Galgani, A. Giorgilli, J.-M. Strelcyn, Meccanica 15 (1981) 21.
[22] J.L. Kaplan, J.A. Yorke, in: H.-O. Peitgen, H.-O. Walter (Eds.), Lecture Notes in Mathematics, Vol. 73, Springer, Berlin, 1979.

[23] E. Barreto, B.R. Hunt, C. Grebogi, J.A. Yorke, Phys. Rev. Lett. 78 (1997) 4561. 